\title{
Stochastic Kinetics and Equilibrium of
}

\section{Nanoconfined Reactions}

Yuri Khodorkovsky[1]*, Leonid Rubinovich ${ }^{[2]}$ and Micha Polak ${ }^{[2]}$

[1] Department of Chemistry, Nuclear Research Center Negev, POB 9001, Beer-Sheva 84190, Israel

[2] Department of Chemistry, Ben-Gurion University of the Negev, Beer-Sheva 84105, Israel

\section{Supporting Information}

S1. Possible breakdown of the mass-action law in relatively large systems

As a demonstration of the effect, biological vesicles containing $\sim 10^{7}-10^{9}$ water molecules ${ }^{1}$ and characterized by the very small equilibrium constant for water dissociation has been chosen. In this system the number of product dissociated molecules is very small on the average. It was predicted ${ }^{2}$ that the breakdown might manifest itself in these systems, although this prediction was criticized in ${ }^{3}$ because biological vesicles are in fact only quasi-confined systems.

* Corresponding author. E-mail: khodoryu@gmail.com, Phone: +972-(0)8-6569349. 


\section{S2. The Gillespie algorithm ${ }^{4}$}

The probability per unit time that $\mu$-th reaction will occur is given by $a_{\mu} \equiv k_{\mu} h_{\mu, x}$, as explained following Equation 2 in the main text. Therefore, the probability per unit time that any reaction will occur is:

$$
a=\sum_{\mu=1}^{M} a_{\mu}
$$

In each step of the algorithm two random numbers, $r_{1}$ and $r_{2}$, drawn from a uniform distribution on a unit interval from 0 to 1 , are generated. The random time $\tau$ that will pass between two successive reactions occurring inside the reaction volume reads,

2

$$
\tau=\frac{1}{a} \ln \frac{1}{r_{1}}
$$

In order to find the identity of the reaction that occurs after time $\tau$, the index $\mu$ must be found for which:

3

$$
\sum_{v=1}^{\mu-1} a_{v}<a r_{2} \leq \sum_{v=1}^{\mu} a_{v}
$$

As an example, for a reversible reaction ( $\mu=1$ or 2 ), the last inequality corresponds to finding whether $a$ $r_{2}$ is smaller or equal to $a_{1}$. If the latter is true - the forward reaction will occur, otherwise - the backward reaction. Further details can be found in Ref. 4 and references therein. 
S3. The absence of confinement effects in unimolecular isomerization reactions

Consider the simplest unimolecular reversible isomerization reaction:

4

$$
\begin{gathered}
k_{1} \\
A \rightleftharpoons B \\
k_{-1}
\end{gathered}
$$

The unimolecular reaction rate constant can be found for simple cases from quantum mechanics ${ }^{4}$. This constant depends on the state of the single molecule, and on the temperature, and does not depend on the volume of the reaction vessel.

The deterministic rate equation for the number of product molecules $x(t)$ is:

5

$$
\frac{d x(t)}{d t}=-\left(k_{1}+k_{-1}\right) x(t)+k_{1} A_{0}-k_{-1} B_{0}
$$

Taking an ensemble average:

6

$$
\frac{d\langle x(t)\rangle}{d t}=-\left(k_{1}+k_{-1}\right)\langle x(t)\rangle+k_{1} A_{0}-k_{-1} B_{0}
$$

As explained in the Theory and Computational Details section of the paper, the same equation is rigorously obtained for $\langle x(t)\rangle$ by means of the Chemical Master Equation (CME). Since this equation for the unimolecular reaction contains only $\langle x(t)\rangle$, its solution is the same as for the deterministic kinetics.

Setting the time derivative in Equation 6 to zero gives for the equilibrium number of reacted molecules: 


$$
\langle x\rangle_{e q}=\frac{k_{1} A_{0}-k_{-1} B_{0}}{k_{1}+k_{-1}},
$$

With $\langle A\rangle_{e q}=A_{0}-\langle x\rangle_{e q}$ and $\langle B\rangle_{e q}=B_{0}+\langle x\rangle_{e q}$, the familiar result for the equilibrium constant, $K_{T L}$, from the law of mass action, is obtained:

8

$$
K_{s t o c}=\frac{\langle B\rangle_{e q}}{\langle A\rangle_{e q}}=\frac{k_{1}}{k_{-1}}=K_{T L}=\frac{B_{e q}}{A_{e q}}
$$

This derivation provides a kinetics-based justification to the absence of the nanoconfinement entropic effect on chemical equilibrium (NCECE) effect for first order reactions ${ }^{5,6}$. The stochastic differential equation for the average number of molecules does not contain nonlinear terms, such as $\left\langle x^{2}(t)\right\rangle$, as is the case for bimolecular reactions. Therefore, the stochastic solution for the mean is indistinguishable from the deterministic solution, and no NC effects appear. This prediction is confirmed in a simulation using the Gillespie algorithm.

\section{S4. Derivations of the "stochastic" equilibrium constant}

a) Stoichiometric exchange reaction

\section{Equilibrium statistical mechanics}

For elementary exchange reaction $A+B \rightleftharpoons C+D$ the partition-function is given for any $N_{0}$ by,

$$
Q=\sum_{N_{C}=0}^{N_{0}} \frac{q_{A} q_{B} q_{C} q_{D}}{N_{A} ! N_{B} ! N_{C} ! N_{D} !} e^{-\left(N_{C}-N_{0}\right) \Delta E / k T},
$$

where $q_{X}(X=A, B, C, D)$ denote the molecular partition functions, and $\Delta E$ is the reaction energy (per product molecule). 
After omitting a microstate-independent pre-factor that does not affect computations of average properties, this equation becomes,

$$
Q=\sum_{N_{C}=0}^{N_{0}} \frac{1}{\left[\left(N_{0}-N_{C}\right) !\right]^{2}\left(N_{C} !\right)^{2}}\left(\frac{q_{C} q_{D}}{q_{A} q_{B}} e^{-\Delta E / k T}\right)^{N_{C}}
$$

Considering first the thermodynamic limit (TL) of very large $N_{0}$, the maximal term in the partition function is highly dominant, and it corresponds to the condition,

$$
K_{T L}=\frac{\left(N_{C}^{T L}\right)^{2}}{\left(N_{0}^{T L}-N_{C}^{T L}\right)^{2}}=\frac{q_{C} q_{D}}{q_{A} q_{B}} e^{-\Delta E / k T}
$$

so,

$$
Q=\sum_{N_{C}=0}^{N_{0}} \frac{1}{\left[\left(N_{0}-N_{C}\right) !\right]^{2}\left(N_{C} !\right)^{2}} K_{T L^{\prime}}^{N_{C}}
$$

and

$$
{\overline{N_{C}}}^{N C}=Q^{-1} \sum_{N_{C}=0}^{N_{0}} \frac{N_{C}}{\left[\left(N_{0}-N_{C}\right) !\right]^{2}\left(N_{C} !\right)^{2}} K_{T L}^{N_{C}}
$$

Case 1. $K_{T L} \gg 1$

Keeping only the two largest contributions,

$$
\begin{gathered}
Q=\frac{1}{\left(N_{0} !\right)^{2}} K_{T L}^{N_{0}}+\frac{1}{\left[\left(N_{0}-1\right) !\right]^{2}} K_{T L}^{N_{0}-1} \text {, and } \\
\bar{N}_{C} N C=\bar{N}_{D}^{N C} \approx \frac{N_{0} \frac{1}{\left(N_{0} !\right)^{2}} K_{T L}^{N_{0}}+\left(N_{0}-1\right) \frac{1}{\left[\left(N_{0}-1\right) !\right]^{2}} K_{T L}^{N_{0}-1}}{{\frac{1}{\left(N_{0} !\right)^{2}}}^{N_{T L}}+\frac{1}{\left[\left(N_{0}-1\right) !\right]^{2}} K_{T L}^{N_{0}-1}}=\frac{N_{0}+\left(N_{0}-1\right) N_{0}^{2} K_{T L}^{-1}}{1+N_{0}^{2} K_{T L}^{-1}} \approx N_{0}\left(1-N_{0} K_{T L}^{-1}\right) .
\end{gathered}
$$

Correspondingly, 


$$
{\overline{N_{A}}}^{N C}={\overline{N_{B}}}^{N C}=N_{0}-{\overline{N_{C}}}^{N C} \approx N_{0}^{2} K_{T L}^{-1},
$$

and so,

$$
K_{\text {stoc }}=\frac{\left({\overline{N_{C}}}^{N C}\right)^{2}}{\left(N_{0}-{\overline{N_{C}}}^{N C}\right)^{2}}=\frac{\left(N_{0}\left(1-N_{0} K_{T L}^{-1}\right)\right)^{2}}{\left(N_{0}^{2} K_{T L}^{-1}\right)^{2}} \approx \frac{K_{T L}^{2}}{N_{0}^{2}}
$$

and

$\log K_{\text {stoc }} \approx 2 \log K_{T L}-2 \log N_{0}$,

which is the same as Equation 16 in the main text.

Case 2. $K_{T L} \ll 1$

Keeping only the two largest contributions,

$$
\begin{aligned}
& Q=\frac{1}{\left(N_{0} !\right)^{2}}+\frac{1}{\left[\left(N_{0}-1\right) !\right]^{2}} K_{T L}, \\
& \bar{N}_{C} N C=\overline{N_{D}} N C=\frac{0 \frac{1}{\left(N_{0} !\right)^{2}}+1 \frac{1}{\left[\left(N_{0}-1\right) !\right]^{2}} K_{T L}}{\frac{1}{\left(N_{0} !\right)^{2}}+\frac{1}{\left[\left(N_{0}-1\right) !\right]^{2}} K_{T L}} \approx N_{0}^{2} K_{T L},
\end{aligned}
$$

and,

$$
{\overline{N_{A}}}^{N C}={\overline{N_{B}}}^{N C}=N_{0}-{\overline{N_{C}}}^{N C} \approx N_{0}-N_{0}^{2} K_{T L} .
$$

So,

$$
K_{\text {stoc }}=\frac{\left({\overline{N_{C}}}^{N C}\right)^{2}}{\left(N_{0}-\bar{N}_{C}^{N C}\right)^{2}}=\frac{\left(N_{0}^{2} K_{T L}\right)^{2}}{\left(N_{0}-N_{0}^{2} K_{T L}\right)^{2}} \approx\left(N_{0} K_{T L}\right)^{2} .
$$

and 


$$
\log K_{\text {stoc }} \approx 2 \log K_{T L}+2 \log N_{0}
$$

which is the same as Equation 18 in the main text.

The asymptotic solution of stochastic kinetics

For the exchange reaction the mean number of the reactant $A$ at equilibrium is given by ${ }^{7}$ :

9

$$
\langle A\rangle_{e q}=\frac{\left(A_{0}+C_{0}\right)\left(A_{0}+D_{0}\right)}{K_{T L}\left(B_{0}-A_{0}+1\right)} \frac{2 F_{1}\left(-A_{0}-C_{0}+1,-A_{0}-D_{0}+1 ; B_{0}-A_{0}+2 ; 1 / K_{T L}\right)}{2 F_{1}\left(-A_{0}-C_{0},-A_{0}-D_{0} ; B_{0}-A_{0}+1 ; 1 / K_{T L}\right)},
$$

with $B_{0} \geq A_{0}$, and $2 F_{1}$ is the Gaussian (ordinary) hypergeometric function, defined by:

10

$$
2 F_{1}(a, b ; c ; z)=1+\frac{a b z}{c 1 !}+\frac{a(a+1) b(b+1) z^{2}}{c(c+1)} 2 !+\frac{a(a+1)(a+2) b(b+1)(b+2) z^{3}}{c(c+1)(c+2)}+\ldots
$$

For the special case of $A_{0}=B_{0}=N_{0}$ and $C_{0}=D_{0}=0$, the expressions for $K_{\text {stoc }}$ as a function of $K_{T L}$ are given in Table S1.

Table S1: The exact and the limiting expressions for $K_{\text {stoch }}$ as a function of $K\left(\equiv K_{T L}\right)$ for small initial number of molecules.

\begin{tabular}{|c|c|c|c|}
\hline$N_{0}$ & Exact $K_{\text {stoc }}$ & $K \gg 1$ limit & $K \ll 1$ limit \\
\hline 1 & $K^{2}$ & $K^{2}$ & $K^{2}$ \\
\hline 2 & $\frac{K^{2}(K+2)^{2}}{(2 K+1)^{2}}$ & $\frac{1}{2^{2}} K^{2}$ & $2^{2} K^{2}$ \\
\hline 3 & $\frac{K^{2}\left(K^{2}+6 K+3\right)^{2}}{\left(3 K^{2}+6 K+1\right)^{2}}$ & $\frac{1}{3^{2}} K^{2}$ & $3^{2} K^{2}$ \\
\hline
\end{tabular}


Comparing this Table with Table S2 for the addition reaction reveals major differences including inverse NCECE in the case of the exchange reaction when $K \ll 1$. In order to find general equation for $K_{s t o c}$ in the limit $K_{T L} \gg 1$, the second and higher order terms in the hypergeometric series 10 are neglected. Substitution in Equation 9 gives,

11

$$
\langle A\rangle_{e q}=\langle B\rangle_{e q}=\frac{N_{0}^{2} 1+\frac{\left(1-N_{0}\right)^{2} 1}{2} K_{T L}}{1+\frac{N_{0}^{2}}{K_{T L}}} \approx \frac{N_{0}^{2}}{K_{T L}}
$$

and

12

$$
\langle C\rangle_{e q}=\langle D\rangle_{e q}=N_{0}-\langle A\rangle_{e q} \approx N_{0}
$$

Now, the "stochastic" equilibrium constant reads,

13

$$
K_{s t o c} \approx\left(\frac{K_{T L}}{N_{0}}\right)^{2} .
$$

This result corresponds to the statistical mechanical result given above, to Equation 16 in the main text, and to the addition reaction results for this limit.

b) Stoichiometric addition reaction

Equilibrium statistical mechanics 
For the elementary addition reaction $A+B \rightleftharpoons C$ the canonical-ensemble partition function from Equation (3) in Ref. 8 reads,

$$
Q=\sum_{N_{C}=0}^{N_{0}} \frac{1}{\left[\left(N_{0}-N_{C}\right) !\right]^{2} N_{C} !}\left(\frac{K_{T L, m o l a r}}{10^{3} V N_{\text {Avogadro }}}\right)^{n_{C}} .
$$

The equilibrium constant in terms of molarities, $K_{T L, m o l a r}$, and $K_{T L}$, in terms of concentrations, are related as, $K_{T L, \text { molar }}=10^{3} N_{\text {Avogadro }} K_{T L}$, so the partition function is given by,

$$
Q=\sum_{N_{C}}^{N_{0}}=0 \frac{1}{\left[\left(N_{0}-N_{C}\right) !\right]^{2} N_{C} !}\left(\frac{K_{T L}}{V}\right)^{N_{C}}
$$

and the average number of product molecules can be written as,

$$
{\overline{N_{C}}}^{N C}=Q^{-1} \sum_{N_{C}=0}^{N_{0}} \frac{N_{C}}{\left[\left(N_{0}-N_{C}\right) !\right]^{2} N_{C} !}\left(\frac{K_{T L}}{V}\right)^{N_{C}}
$$

Now, keeping only the two largest contributions to the sums above, which is valid for the case of $K_{T L} \gg 1$ (e.g., exothermic reaction at low temperatures), it follows that

$$
\begin{aligned}
& Q=\frac{1}{N_{0} !}\left(\frac{K_{T L}}{V}\right)^{N_{0}}+\frac{1}{\left(N_{0}-1\right) !}\left(\frac{K_{T L}}{V}\right)^{N_{0}-1} \\
& \bar{N}_{C} N C \approx \frac{N_{0} \frac{1}{N_{0} !}\left(\frac{K_{T L}}{V}\right)^{N_{0}}+\left(N_{0}-1\right) \frac{1}{\left(N_{0}-1\right) !}\left(\frac{K_{T L}}{V}\right)^{N_{0}-1}}{\frac{1}{N_{0} !}\left(\frac{K_{T L}}{V}\right)^{N_{0}}+\frac{1}{\left(N_{0}-1\right) !}\left(\frac{K_{T L}}{V}\right)^{N_{0}-1}} \approx N_{0}\left[1-\left(\frac{K_{T L}}{V}\right)^{-1}\right], \\
& c_{C_{N C}}=\frac{N_{0}}{V}\left[1-\left(\frac{K_{T L}}{V}\right)^{-1}\right] \approx \frac{N_{0}}{V}, \text { and } \\
& c_{A_{N C}}=c_{B_{N C}}=\frac{N_{0}-\bar{N}_{C}^{N C}}{V}=\frac{1}{V}\left\{N_{0}-N_{0}\left[1-\left(\frac{K_{T L}}{V}\right)^{-1}\right]\right\}=\frac{N_{0}}{V}\left(\frac{K_{T L}}{V}\right)^{-1} .
\end{aligned}
$$

So, 


$$
K_{\text {stoc }}=\frac{c_{C_{N C}}}{c_{B N C} c_{B N C}}=\frac{V}{N_{0}}\left(\frac{K_{T L}}{V}\right)^{2}=\frac{1}{N_{0} V}\left(K_{T L}\right)^{2},
$$

or,

$$
\log K_{\text {stoc }}=2 \log K_{T L}-\log \left(N_{0} V\right),
$$

If the volume units are chosen so that the initial concentration $c_{0}=\frac{N_{0}}{V}=1$, then the following expression is obtained,

$$
\log K_{\text {stoc }}=2 \log K_{T L}-2 \log N_{0} .
$$

The asymptotic solution of the stochastic kinetics

For the addition reaction $A+B \rightleftharpoons C$ the corresponding number of $A$ molecules at equilibrium is given by?:

14

$$
\langle A\rangle_{e q}=\frac{A_{0}+C_{0}}{K_{T L}^{\prime}\left(B_{0}-A_{0}+1\right)} \frac{1 F_{1}\left(-A_{0}-C_{0}+1 ; B_{0}-A_{0}+2 ;-1 / K_{T L}^{\prime}\right)}{1 F_{1}\left(-A_{0}-C_{0} ; B_{0}-A_{0}+1 ;-1 / K_{T L}^{\prime}\right)},
$$

with $B_{0} \geq A_{0}, 1 F_{1}$ is the confluent hypergeometric function, defined by the infinite series:

15

$$
1 F_{1}(a ; b ; z)=1+\frac{a z}{b 1 !}+\frac{a(a+1) z^{2}}{b(b+1) 2 !}+\frac{a(a+1)(a+2) z^{3}}{b(b+1)(b+2) 3 !}+\ldots
$$

and the equilibrium constant defined in terms of molecular numbers is $K_{T L}^{\prime}=\frac{k_{1}}{k_{-1}}=\left(\frac{C}{A B}\right)_{e q}$. The stochastic equilibrium constant, $K_{s t o c}^{\prime}=\left(\frac{\langle C\rangle}{\langle A\rangle\langle B\rangle}\right)_{e q}$, can be now found using $\langle B\rangle_{e q}=B_{0}-\left(A_{0}-\langle A\rangle_{e q}\right)$ and $\langle C\rangle_{e q}=C_{0}+\left(A_{0}-\langle A\rangle_{e q}\right)$ 
For the special case of $A_{0}=B_{0}=N_{0}$ and $C_{0}=0$, the expressions for $K_{s t o c}^{\prime}$ as a function of $K_{T L}^{\prime}$ are given in Table S2.

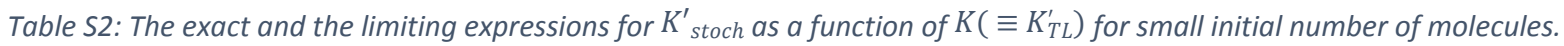

\begin{tabular}{|c|c|c|c|}
\hline$N_{0}$ & Exact $K_{\text {stoch }}^{\prime}$ & $K \gg 1$ limit & $K \ll 1$ limit \\
\hline 1 & $K(K+1)$ & $K^{2}$ & $K$ \\
\hline 2 & {$\left[1-2\left(\frac{K}{2 K+1}\right)^{2}\right] K(K+1)$} & $\frac{1}{2} K^{2}$ & $K$ \\
\hline 3 & $\frac{K\left(2 K^{2}+4 K+1\right)\left(6 K^{3}+18 K^{2}+9 K+1\right)}{\left(6 K^{2}+6 K+1\right)^{2}}$ & $\frac{1}{3} K^{2}$ & $K$ \\
\hline
\end{tabular}

As previously mentioned, and as explained in the main text, the addition reaction reveals no NCECE when $K \ll 1$, as seen in the Table, whereas for $K \gg 1$ the NCECE decreases with $N_{0}$ increase. In order to find the general equation for $K_{\text {stoc }}^{\prime}$ in this limit, the second and higher order terms in the hypergeometric series 15 are neglected, obtaining from Equation 14:

16

$$
\langle A\rangle_{e q}=\langle B\rangle_{e q}=\frac{N_{0} 1-\frac{1-N_{0} 1}{2} K_{T L}^{\prime}}{1+\frac{N_{0}}{K_{T L}^{\prime}}} \approx \frac{N_{0}}{K_{T L}^{\prime}}
$$

and 


$$
\langle C\rangle_{e q}=N_{0}-\langle A\rangle_{e q} \approx N_{0}
$$

So that the "stochastic" equilibrium constant reads:

18

$$
K_{\text {stoc }}^{\prime} \approx \frac{\left(K_{T L}^{\prime}\right)^{2}}{N_{0}} .
$$

After defining the equilibrium constants in terms of concentrations, $K_{s t o c}=V K_{s t o c}^{\prime}$ and $K_{T L}=V K_{T L}^{\prime}$, multiplying Equation 18 by $V^{2}$ and choosing a constant initial concentration as $c_{0}=N_{0} / V=1$,

19

$$
K_{s t o c} \approx\left(\frac{K_{T L}}{N_{0}}\right)^{2}
$$

This corresponds to the statistical mechanical result given above and to Equation 16 in the main text.

\section{References}

[1] A. Koudriavtsev, R. Jameson and W. Linert, The Law of Mass Action, Berlin: Springer-Verlag, 2001, pp. 99-105.

[2] L. A. Blumenfeld, A. Y. Grosberg and A. N. Tikhonov, J. Chem. Phys., vol. 95, pp. 7541-7547, 1991.

[3] A. V. Sokirko, J. Chem. Soc., Faraday Trans., vol. 90, pp. 2353-2358, 1994.

[4] D. T. Gillespie, A. Hellander and L. R. Petzold, "Perspective: stochastic algorithms for chemical kinetics," J. Chem. Phys., vol. 138, p. 170901, 2013.

[5] T. L. Hill, Thermodynamics of Small Systems, New York: Dover: Mineola, 2002.

[6] M. Polak and L. Rubinovich, "Nanochemical equilibrium involving a small number of molecules: a prediction of a distinct confinement effect," Nano Lett., vol. 8, pp. 3543-3547, 2008. 
[7] I. G. Darvey, B. W. Ninham and P. J. Staff, "Stochastic models for second-order chemical reaction kinetics. The equilibrium state," J. Chem. Phys., vol. 45, no. 6, p. 2145, 1966.

[8] L. Rubinovich and M. Polak, "Remarkable NanoConfinement Effects on Equilibrated Reactions: Statistical-Mechanics Modeling Focused on Ir Dimerization Beneath Surface Sites in Pd-Ir Nanoparticles," Topics in Catalysis, vol. 61, pp. 1237-1246, 2018. 